\title{
A SETAR Model for Canadian GDP: Non-linearities and Forecast Comparisons
}

\author{
Hui Feng and Jia Liu \\ Department of Economics, University of Victoria, B.C., Canada \\ October, 2002
}

\begin{abstract}
In this paper we investigate the forecasting performance of the non-linear time series SETAR model by using Canadian GDP data from 1965 to 2000. Besides the with-insample fit, the forecasting performance of a standard linear ARIMA model for the same sample has also been generated for comparative purposes. Two forecasting methods, 1step-ahead and multi-step-ahead forecasting are compared for each type of model.
\end{abstract}

Key Words: $\quad$ Threshold model, non-linearities, forecasting, ARIMA model

JEL Classifications: C22, C53

Author Contact: Hui Feng

Department of Economics

University of Victoria, P.O. Box 1700 STN CSC

Victoria, B.C., Canada, V8W 2Y2

E-mail: hui@uvic.ca; FAX (250) 721 6214; Phone: (250) 4724418 


\section{Introduction}

In recent years, more and more attention has been given to modelling and forecasting the non-linearity in various macroeconomic series, such as GDP and the unemployment rate. A number of models have been applied, such as the GARCH, ARIMA, some regimeswitching models (the threshold and Markov switching autoregressive models) and so on. Among them, the non-linear regime-switching models are comparatively more popular. However, from the forecasting point of view, there appears to be no clear conclusion as to whether allowing for non-linearity leads to an improvement in forecast performance (see, e.g., De Gooijer and Kumar, 1992). In this paper, we investigate the forecast performance of a nonlinear time series model, which has been proposed in the literature for modeling GDP, exchange rates, and other time series data - the Self-Exciting Threshold Autoregressive (SETAR) model. SETAR models have been employed to analyze U.S. GNP and U.K. GDP data, (see, e.g., Potter, 1995; Peel and Speight, 1995). However, no such attention has been paid to Canadian GDP data. In this paper, we suggest that Canadian expenditure based real GDP is generated by a nonlinear SETAR model. After fitting the preferred SETAR model to the data, we compare the forecast performance of this model with that of the linear ARIMA ${ }^{1}$ model, in order to judge the out-of-sample forecasting ability of the nonlinear SETAR model.

The remainder of this paper is organized as follows. Section II briefly describes the nonlinear regime-switching SETAR model. Section III introduces the out-of-sample forecasting method that we use. Section IV shows the model selection process and the within sample fits of the SETAR model and the ARIMA model. Section V presents the out-of-sample forecasting performance of both models, and Section VI offers a summary of our findings.

\section{Model Description}

The idea of multi-regime forecasting models dates back at least to Bacon and Watts (1971). Tong (1978) initially proposed the Threshold Autoregressive (TAR) model, which assumes that the regime that occurs at time $t$ can be determined by an observable 
variable $q_{t}$ relative to a threshold value, which we denote as $c$. The SETAR model assumes that the threshold variable $q_{t}$ is chosen to be the lagged value of the time series itself. That is to say, if $\mathrm{y}$ is the series being modeled, then $q_{t}=y_{t-d}$ for a certain integer $d>0$. Hence, the SETAR model is linear within a regime, but liable to move between regimes as the process crosses the threshold: see Tong (1990) and Hansen (1997, 2000). When there are only two regimes, and the process is a $p$-th order autoregression in each regime, we convey this information more briefly by writing $\operatorname{SETAR}(2 ; p ; p)$, and algebraically:

$y_{t}=\left(\phi_{0,1}+\phi_{1,1} y_{t-1}+\cdots+\phi_{p, 1} y_{t-p}\right) I\left[y_{t-d} \leq c\right]+\left(\phi_{0,2}+\phi_{1,2} y_{t-1}+\cdots+\phi_{p, 2} y_{t-p}\right) I\left[y_{t-d}>c\right]+\varepsilon_{t}$

where $I[\mathrm{~A}]$ is an indicator function with $I[\mathrm{~A}]=1$ if the event $\mathrm{A}$ occurs and $I[\mathrm{~A}]=0$ otherwise. In particular, in the 2-regime SETAR model, $y_{t}$ will be estimated within the first regime if the value of $y_{t-d}$ is smaller or equal to the threshold variable $c$, otherwise, $y_{t}$ will be estimated within the second regime. More compactly, this SETAR model can be rewritten as,

$$
y_{t}=\phi_{1}^{\prime} x_{t} I\left[y_{t-1} \leq c\right]+\phi_{2}^{\prime} x_{t} I\left[y_{t-1}>c\right]+\varepsilon_{t}
$$

where $\phi_{j}=\left(\phi_{0, j}, \phi_{1, j}, \cdots, \phi_{p, j}\right)^{\prime}, j=1,2$, and $x_{t}=\left(1, y_{t-1}, \cdots, y_{t-p}\right)^{\prime}$. Note that when the threshold variable $c$ is fixed, the model is linear in the remaining parameters. Estimators of the parameters can be obtained easily by OLS if we can find the appropriate threshold variable $c$. In the context of the SETAR models we restrict the candidate threshold variable $c$ to be the lagged endogenous variable $y_{t-d}$ for a positive integer $d$. The estimators of the nuisance parameters, namely the threshold value $c$ and the lag-length $d$, can be determined by minimizing the residual variance, or optimizing some other such criterion, among a reasonable range of choices of $c$ and $d$ (say, $c \in \mathrm{C}$ and $d \in\left\{1, \cdots, d^{*}\right\}$ for some upper bound $d^{*}$ ). The set of allowable threshold values, $\mathrm{C}$, should be such that each regime contains enough observations for the estimator defined above to produce reliable estimates of the autoregressive parameters. A popular choice of $\mathrm{C}$ is to allow each regime to have at least a fraction $\pi$ of the observations, that is, 


$$
C=\left\{c \mid y_{([\pi(n-1)])} \leq c \leq y_{([(1-\pi)(n-1)])}\right\}
$$

A safe choice for this fraction appears to be 0.15 (Franses and van Dijk, 2000). For an optimization criterion we follow standard practice and use the Akaike Information Criterion (AIC). Then identification of the appropriate threshold variable $c$ and lag order $d$ can be chosen from the model that will minimize the AIC value. Tong (1990) defines the AIC for a 2-regime SETAR model as the sum of the AICs for the AR models in the two regimes, which is:

$$
\operatorname{AIC}\left(p_{1}, p_{2}\right)=n_{1} \ln \hat{\sigma}_{1}^{2}+n_{2} \ln \hat{\sigma}_{2}^{2}+2\left(p_{1}+1\right)+2\left(p_{2}+1\right)
$$

where $\hat{\sigma}_{j}^{2}, \mathrm{j}=1,2$, is the variance of the residuals in the $j$-th regime and in our case we set $p_{1}=p_{2}=p$.

\section{Methods for Out-of-Sample Forecasting}

The main purpose of this paper is to explore the out-of-sample forecast performance of the non-linear SETAR model when applied to Canadian real GDP data. SETAR models have been successfully used to forecast certain biological or physical processes, such as the Canadian lynx data and Wolf's sunspot numbers (see Tong). They have also been applied to a number of economic and financial data. For example, Kräger and Kugler (1993), Peel and Speight (1994) and Chappell et al. (1996) apply such models to the foreign exchange market; Tiao and Tsay (1994) and Potter (1995) apply them to the US GNP data; and Montgomery, Zarnowitz et al. (1998) and Rothman (1998) apply them to unemployment rates.

Computing point forecasts from non-linear models is much more complicated than from a linear model. The optimal one-step-ahead forecast could be denoted as: 


$$
\hat{y}_{t+\mid t}=\mathrm{E}\left[y_{t+1} \mid \Omega_{t}\right]=F\left(x_{t} ; \phi\right)
$$

where $\hat{y}_{t+1}$ is the forecast value for the time $(\mathrm{t}+1)$, and the $\Omega_{\mathrm{t}}$ is the history of the time series up to and including the observation at time $t . F\left(x_{t} ; \phi_{j}\right)$ is the non-linear function that represents the SETAR model in equation (1).

When the forecast horizon is longer than one period, things become more complicated. The optimal 2-step-ahead forecast can be obtained as,

$$
\hat{y}_{t+2 \mid t}=\mathrm{E}\left[y_{t+2} \mid \Omega_{t}\right]=E\left[F\left(x_{t+1} ; \phi\right) \Omega_{t}\right]
$$

In general, the linear conditional expectation operator $\mathrm{E}$ can not be interchanged with the nonlinear operator $\mathrm{F}$, that is,

$$
\hat{y}_{t+2 \mid t}=\mathrm{E}\left[y_{t+2} \mid \Omega_{t}\right]=E\left[F\left(x_{t+1} ; \phi\right) \mid \Omega_{t}\right] \neq F\left(E\left[y_{t+1} \mid \Omega_{t}\right] ; \phi\right)=F\left(\hat{y}_{t+1 \mid t} ; \phi\right)
$$

As a result, the general way of calculating the multi-step-ahead forecast is somewhat biased $^{2}$. The optimal $h$-step-ahead forecast is computed as (see, Franses and van Dijk, 2000):

$$
\hat{y}_{t+h \mid t}=\mathrm{E}\left[y_{t+h} \mid \Omega_{t}\right]=F\left(x_{t+h-1} ; \phi\right)
$$

Notice that the difference between the 1-step-ahead forecast and the multi-step-forecast is that the first one is computed using all the original data, but the latter one is computed using not only the original data but also the forecast values of the previous $h-1$ periods.

Several methods for multi-step-ahead forecasting have been developed in recent years. These include one suggested by Tong (1995), which is based on the ChapmanKolmogorov relation and requires computer-intensive sequences of numerical integration; a Monte Carlo method that is discussed by Tong (1995), Tiao and Tsay 
(1994) and Clements and Smith (1997); and the Normal Forecast Error (NFE) method proposed by Al-Qassam and Lane (1989) and De Gooijer and De Bruin (1998). Lin and Granger (1994) have found that the Monte Carlo and bootstrap methods are comparatively favorable to the other methods. In our research, we have a total of 160 observations and 5 observations are kept for the evaluation of out-of-sample forecasts. As a result, to employ the Monte Carlo or bootstrap methods for 5-step-ahead forecast is quite computer-intensive. Hence, we explore the forecasting ability of our nonlinear SETAR model by using the straightforward multi-step-ahead forecasts as well as the onestep-ahead forecasts in terms of simply goodness of fit criteria, and a formal test proposed by Diebold and Mariano (1995).

\section{Model Selection}

In this paper, we use quarterly Canadian real GDP data measured in 1992 constant prices from 1961Q1 to 2000Q4. In order to identify the appropriate threshold variable and lag orders, we choose the model that minimizes the AIC value. Here, we are going to use the level series to fit in the model; the unit root has been neglected. The reason for this is that recent research suggests that unit root tests have low power in discriminating against trend stationary and other alternatives in typical macro-econometric contexts (Christiano and Eichenbaum, 1990; Sowell, 1992). In particular, the power of such tests can be dramatically diminished and a unit root mistakenly identified in the presence of threshold determined regime switching ${ }^{3}$. The data are seasonal unadjusted, and we use seasonal dummy variables to remove the seasonal effect from the data. The AIC results are shown in Table 1.

\section{Table 1: AIC values for SETAR models}

\begin{tabular}{|c|c|c|c|c|c|c|c|c|}
\hline \multirow{2}{*}{$\begin{array}{c}\text { Threshold } \\
\text { Variable }\end{array}$} & \multicolumn{7}{|c|}{$P$} \\
\cline { 2 - 9 } & 1 & 2 & 3 & 4 & 5 & 6 & 7 & 8 \\
\hline$y_{t-1}$ & 6268339 & 5802628 & 5674847 & 5420734 & 4608322 & 4531991 & 4614133 & 4716153 \\
\hline$y_{t-2}$ & 6439843 & 5669398 & 5715459 & 5182881 & 4630382 & 4346529 & 4781011 & 4811686 \\
\hline$y_{t-3}$ & 6277664 & 5760481 & 5711611 & 5403410 & 4659633 & 4517147 & 4716370 & 4688142 \\
\hline$y_{t-4}$ & 6525484 & 5958005 & 5900524 & 5499211 & 4794733 & 4574406 & 4847132 & 4728495 \\
\hline$y_{t-5}$ & 6671515 & 6041598 & 5997666 & 5511914 & 4583975 & 4701486 & 4907198 & 4965573 \\
\hline$y_{t-6}$ & 6446246 & 5877706 & 5828359 & 5582834 & 4651675 & 4493038 & 4715415 & 4712743 \\
\hline
\end{tabular}


Applying the method of choosing a proper model by minimizing the AIC value, we find the most appropriate model in this case should be $\operatorname{SETAR}(2,6,6)$ with the threshold variable $y_{t-2}$. In order to compare the non-linear SETAR model with a linear model, we choose the ARIMA model to fit the data. Considering the seasonal effects, we add seasonal autoregressive (AR) term and moving average (MA) terms to the model ${ }^{4}$. The same criteria for choosing the proper model still apply here, that is, the best ARIMA model should be the one that gives the smallest AIC value, provided that residuals are also free of any autocorrelation.

The maximum lags in the AR and MA process were set to eight. The number of seasonal $\mathrm{AR}$ and seasonal MA terms ranged from one to. Table 2 shows the results for the ARIMA models. Hence, the ARIMA $(5,0,3)$ with 2 seasonal AR factors and 2 seasonal MA factors is our preferred specification.

Table 2: 10 smallest AIC values for ARIMA models

\begin{tabular}{|c|c|c|c|c|}
\hline RANK & ARIMA specifics & Seasonal AR term & Seasonal MA term & AIC \\
\hline 1 & ARIMA $(5,0,3)$ & SAR $(2)$ & SMA (2) & 14.903 \\
\hline 2 & ARIMA $(2,0,4)$ & SAR $(1)$ & SMA (1) & 14.913 \\
\hline 3 & ARIMA $(6,0,7)$ & SAR $(1)$ & SMA (1) & 14.914 \\
\hline 4 & ARIMA $(4,0,4)$ & SAR (2) & SMA (2) & 14.916 \\
\hline 5 & ARIMA $(5,0,1)$ & SAR (3) & SMA (3) & 14.924 \\
\hline 6 & ARIMA $(5,0,1)$ & SAR (1) & SMA (1) & 14.929 \\
\hline 7 & ARIMA (7,0,4) & SAR (2) & 14.931 \\
\hline 8 & ARIMA $(3,0,4)$ & SAR $(1)$ & SMA (1) & 14.933 \\
\hline 9 & ARIMA $(6,0,0)$ & SAR (1) & SMA (1) & 14.937 \\
\hline 10 & ARIMA (3,0,3) & SAR (1) & SMA (1) & 14.937 \\
\hline
\end{tabular}

*SAR and SMA stand for the seasonal AR and MA specification in SHAZAM

The topic of interest here is how well the preferred SETAR model and the preferred ARIMA model fit the data and how well both models perform at forecasting. For this purpose, we use the mean absolute percentage error (MAPE):

$$
\text { MAPE }=\left[1 / n \sum_{i=1}^{n} a b s\left(\left(\hat{y}_{t}-y_{t}\right) / y_{t}\right)_{t}\right]^{*} 100
$$

where $\mathrm{n}$ is the number if forecast periods, and $\hat{y}_{t}$ is the forecast value for $\mathrm{y}_{\mathrm{t}}$. 
However, the choice of evaluation criteria is also very important in the sense that the forecast performance depends on them. As noted by Dacco and Satchell (1999), who demonstrate that even if time series are generated according to a regime-switching process, the MAPE of a linear model can be smaller than the MAPE of the true nonlinear model. An alternative way of evaluating the forecast performance is using the root mean square prediction error (RMSPE):

$$
\mathrm{RMSPE}=\sqrt{1 / n \sum_{i=1}^{n}\left[\left(\hat{y}_{t}-y_{t}\right) / y_{t}\right]^{2}} * 100
$$

\section{Table 3: The within-sample MAPE and RMSPE for the two models}

\begin{tabular}{|c|c|c|}
\hline & MAPE & RMSPE \\
\hline SETAR & 0.8754 & 1.1084 \\
\hline ARIMA & 0.9694 & 1.1914 \\
\hline
\end{tabular}

According to our results shown in the Table 3 above, the preferred SETAR model is better than the preferred ARIMA model over the sample period $(n=155)$. Overall, the SETAR model gives better within-sample performance than the ARIMA model. Figure 1 shows the percentage errors of the two models for every observation within the sample. The SETAR model tends to be relatively more stable than the ARIMA model, in the sense that the range of the percentage error for SETAR model is smaller than that of the ARIMA. 
Figure 1: Two models' within sample percentage error

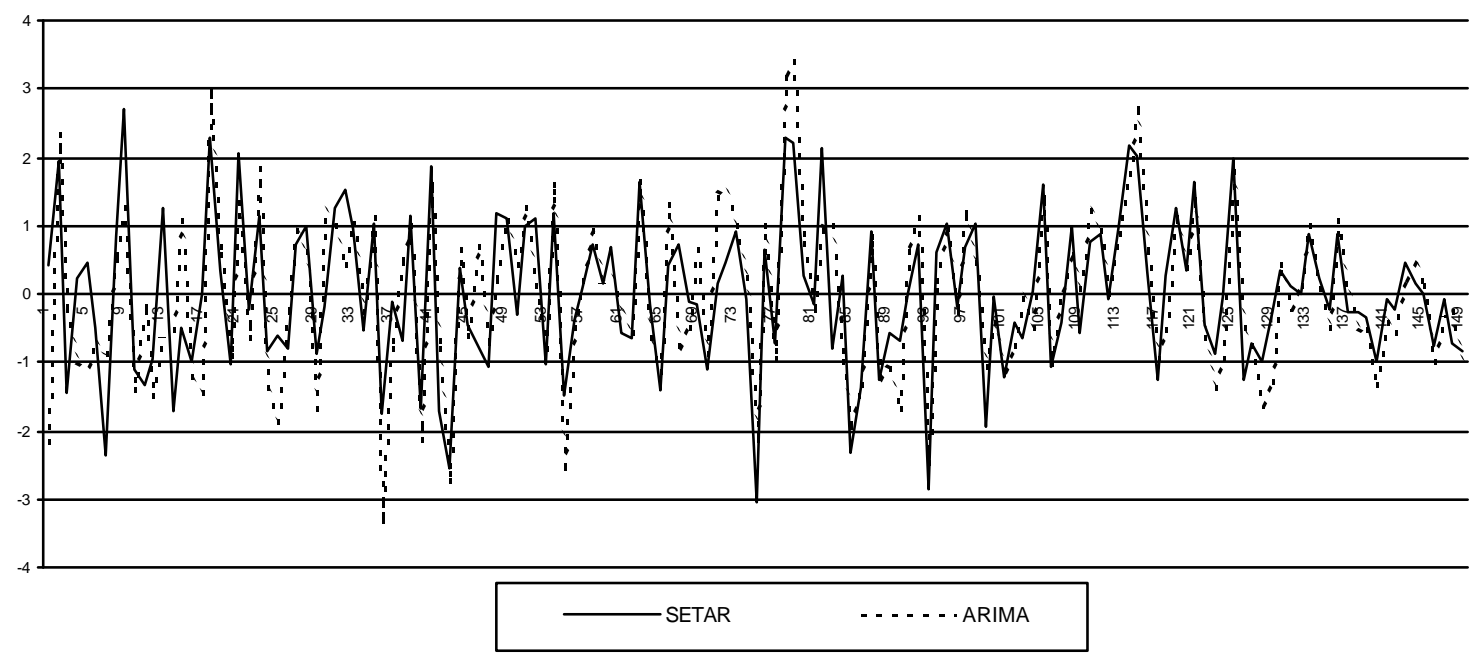

\section{Out-of-sample forecasting performance}

\section{The forecasting performance using the Multi-step-ahead method}

In this section, we check the out-of-sample forecast performance of the two models. Table 4 shows their out-of-sample forecast MAPE, RSMPE, and also the individual forecast percentage error using the multi-step-ahead forecasting procedure. That is, in periods 2 to 5, previous periods' forecasts are used as part of the forecasting equation. Notice here that we are doing a non-adaptive scheme: over all the forecasting periods, we do not change the specification of the preferred model every time.

Table 4: Forecast Percentage Error, MAPE and RSMPE using the multi-step-ahead Forecast Method

\begin{tabular}{|c|c|c|c|c|c|c|c|}
\hline & \multicolumn{5}{|c|}{ Forecast percentage error } & \multirow{2}{*}{} & \multirow{2}{*}{} \\
\cline { 2 - 6 } & $1999 \mathrm{Q} 4$ & $2000 \mathrm{Q} 1$ & $2000 \mathrm{Q} 2$ & $2000 \mathrm{Q} 3$ & $2000 \mathrm{Q} 4$ & MAPE* & RMSPE* \\
\hline SETAR & -0.087 & -0.154 & -1.114 & -1.392 & -0.447 & 0.6388 & 0.8258 \\
\hline ARIMA & -0.233 & -0.501 & -1.469 & -1.847 & -1.131 & 1.0362 & 1.4308 \\
\hline
\end{tabular}

* The MAPE and RMSPE are computed over the five forecasting periods, from 1999Q1-2000Q4 
For each the five forecasting periods, both the SETAR and ARIMA models tend to under-forecast the original data, which may reflect the deterministic trend in the data. However, the MAPE and RMSPE forecasting criteria strongly support the SETAR model. For every forecasting period, we can see that the SETAR model dominates the ARIMA model in the respect of the forecast percentage errors.

Figure 2 below gives us a very clear picture of this. It shows the multi-step-ahead forecasted values from both models compared with the original GDP series. Notice, that both models underestimate the data series. However, both models interpret the structure of the series quite well for the two turning points from the original series are well captured by the two forecasted series respectively. We can see that the forecasts of the SETAR model are always closer to our original data than that of the ARIMA model.

Figure 2: Original GDP data and predicted value from SETAR and ARIMA model using the multi-step-ahead Forecast Method

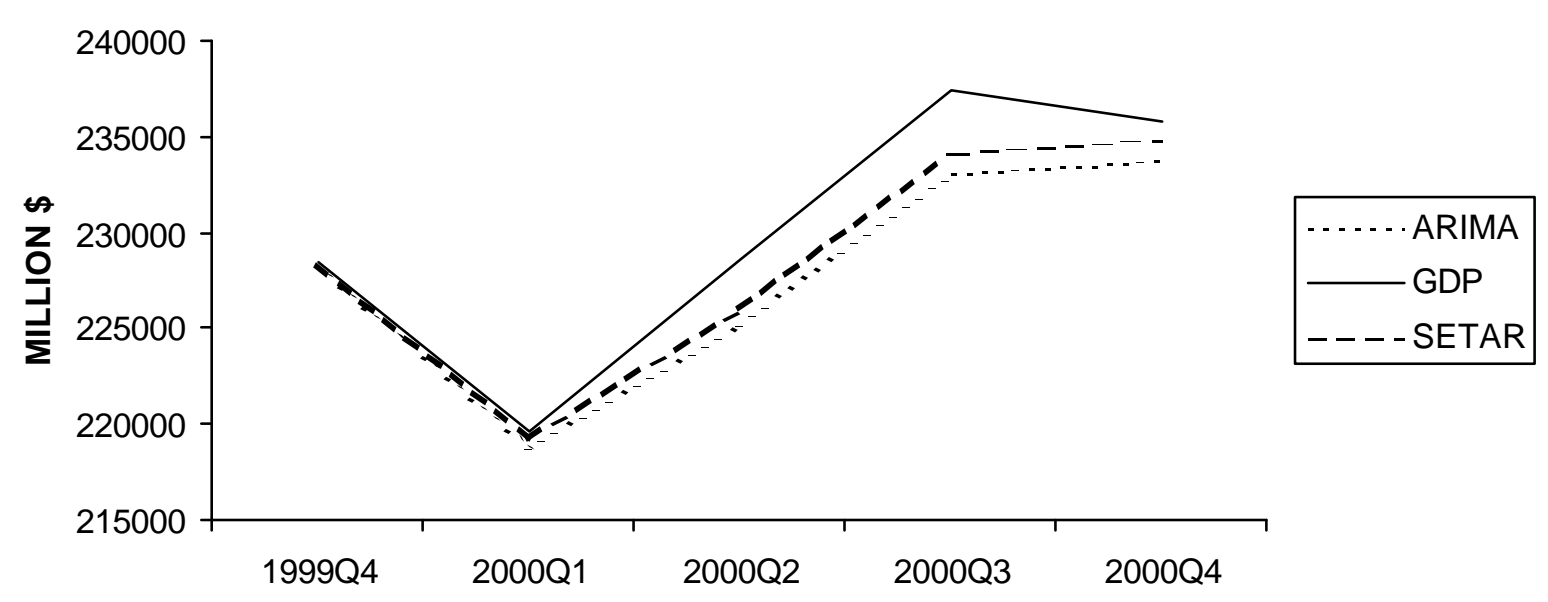

Finally, we have used the "S-test" proposed by Diebold and Mariano (1995) to test the hypothesis that there is no difference between the forecast performances of the two models. The test statistic is:

$$
S=\left(\frac{m}{4}\right)^{-\frac{1}{2}}\left[\sum_{i=1}^{12} d_{i}-\frac{m}{2}\right]
$$


where $m$ is the number of the forecast periods; $d_{i}=1$ if the squared prediction error for the SETAR model exceeds that for the ARIMA model, and is zero otherwise. Under the null hypothesis of equal performance, $S$ is asymptotically standard normal. Using the information in Table 4, $S=-2.2$, so we conclude that the SETAR model out-performs the ARIMA model, at least at the $5 \%$ level of significance.

\section{The forecasting performance using the 1-period-ahead forecast method}

As we mentioned previously, the multi-step-ahead forecasts tends to give us biased prediction after the first forecasting period. One-step-ahead forecasting is different from multi-step-ahead forecasting in the way that it uses the actual data to predict for every forecasting period. Table 5 shows the forecast results for one-step-ahead recursive forecasting, so called because we re-estimate the model every time that we add one more period data in to the sample series.

Table 5: Forecast Percentage Error for one-step-ahead Forecasts, MAPE and RSMPE

\begin{tabular}{|c|c|c|c|c|c|c|c|}
\hline & \multicolumn{5}{|c|}{ Forecast percentage error } & \multirow[b]{2}{*}{ MAPE* } & \multirow[b]{2}{*}{ RMSPE* $^{*}$} \\
\hline & 1999Q4 & 2000Q1 & $2000 Q 2$ & $2000 Q 3$ & $2000 \mathrm{Q} 4$ & & \\
\hline SETAR & -0.087 & -0.055 & -0.935 & -0.207 & 1.277 & 0.5122 & 0.6495 \\
\hline ARIMA & -0.233 & -0.079 & -0.858 & -0.277 & 1.111 & 0.5116 & 0.7154 \\
\hline
\end{tabular}

* The MAPE and RMSPE are computed over the five forecasting periods, from 1999Q1-2000Q4

From the forecasting percentage errors, we can see that in the third and fifth forecasting periods, the ARIMA model is slightly better than the SETAR model. However, for the other three cases, the SETAR model is better. In addition, the forecast RMSPE of the SETAR model is 0.6495 , and for the ARIMA model is 0.7154 . However, the MAPE for the SETAR model is slightly worse than the ARIMA model. In this case the $S$-test of Diebold and Mariano is especially helpful. Using the information in Table 5 we obtain $S$ $=-0.447$, and so there is no significant difference between the forecasting performances of the SETAR and ARIMA models. 
Figure 3 demonstrates the five one-step-ahead forecasting values for both models, compared with the original GDP series. From this chart, we can clearly observe that the forecasted values of both models cross each other. Neither of them dominates the other. In contract with the multi-step-ahead forecasting method, which gives us underestimations in both cases, the results from the 1-step-ahead forecasting method here is: this one-step-ahead forecasting method are mixed. This may suggest that the autocorrelation between the forecasting periods may be smaller in this case than in the multi-step-ahead case. Both models capture the turning points in the data very well.

Figure 3: Original GDP data and predicted value from the SETAR and the ARIMA model using 1-step-ahead Forecasting Method

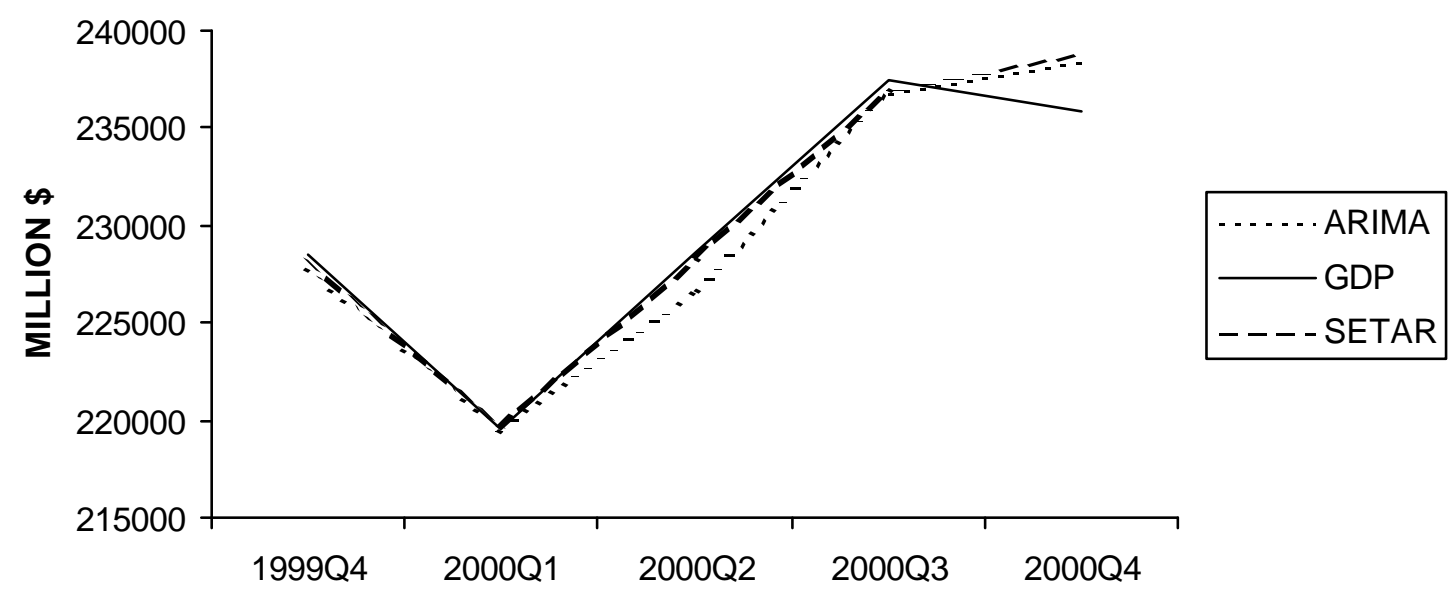


Figure 4: The results between the 1-step-ahead and the multi-step-ahead forecasts using the SETAR model

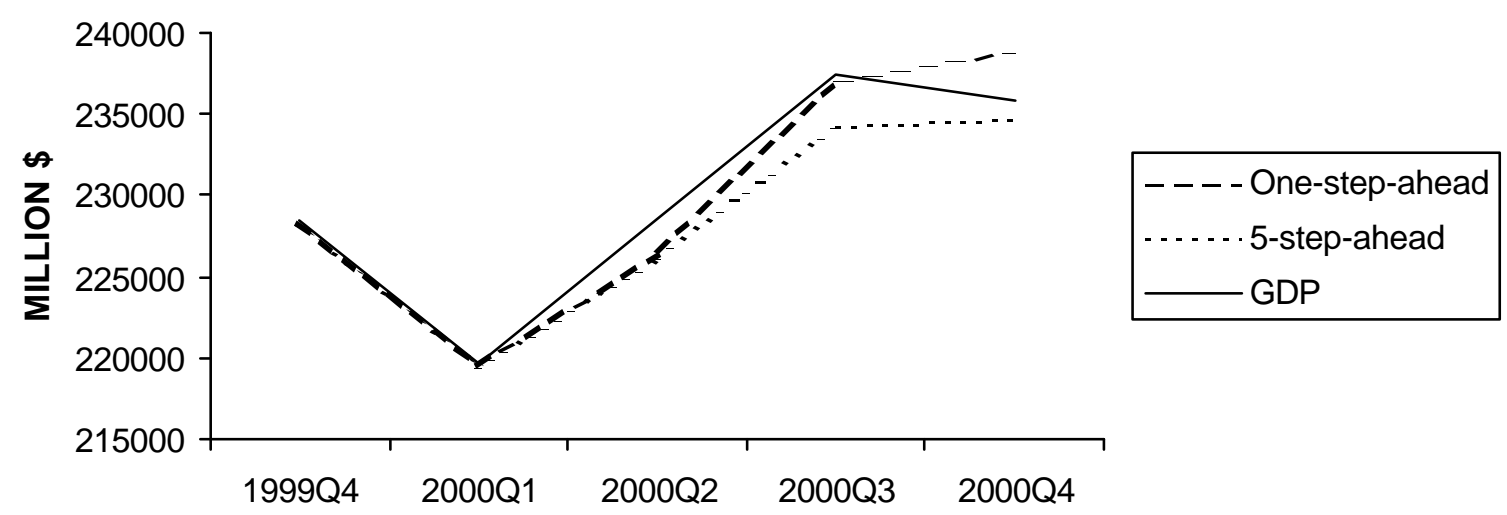

Comparing the forecasting results of these two different forecasting methods in figure 4, we can see that the MAPE and RMSPE are all smaller than those of the multi-step-ahead forecast. It would be too premature to conclude that the 1-step-ahead forecast is better than the multi-step-ahead forecast. The reasons are as follows. First, the two forecasting results we have obtained are based on different data information. We have indicated before that for the 1-step-ahead forecast we are using the recursive scheme, which means that the sample size of the model is increasing every time, while in the multi-step-ahead forecast case we are always using the same sample size. Thus, in this sense the two forecasting methods are non-comparable because of the unequal data information. Second, in the real day life, the multi-step-ahead forecast may have a wider usage than that of the 1-step-ahead forecast because the second period forecasting using the 1-stepahead forecasting could be happen only when the first forecasting period has been realized. We know that especially for these macroeconomic data, we would like to predict several periods ahead, and not simply the next one period 
Figure 5: Forecasting interval of the ARIMA model and the forecasted values from both models

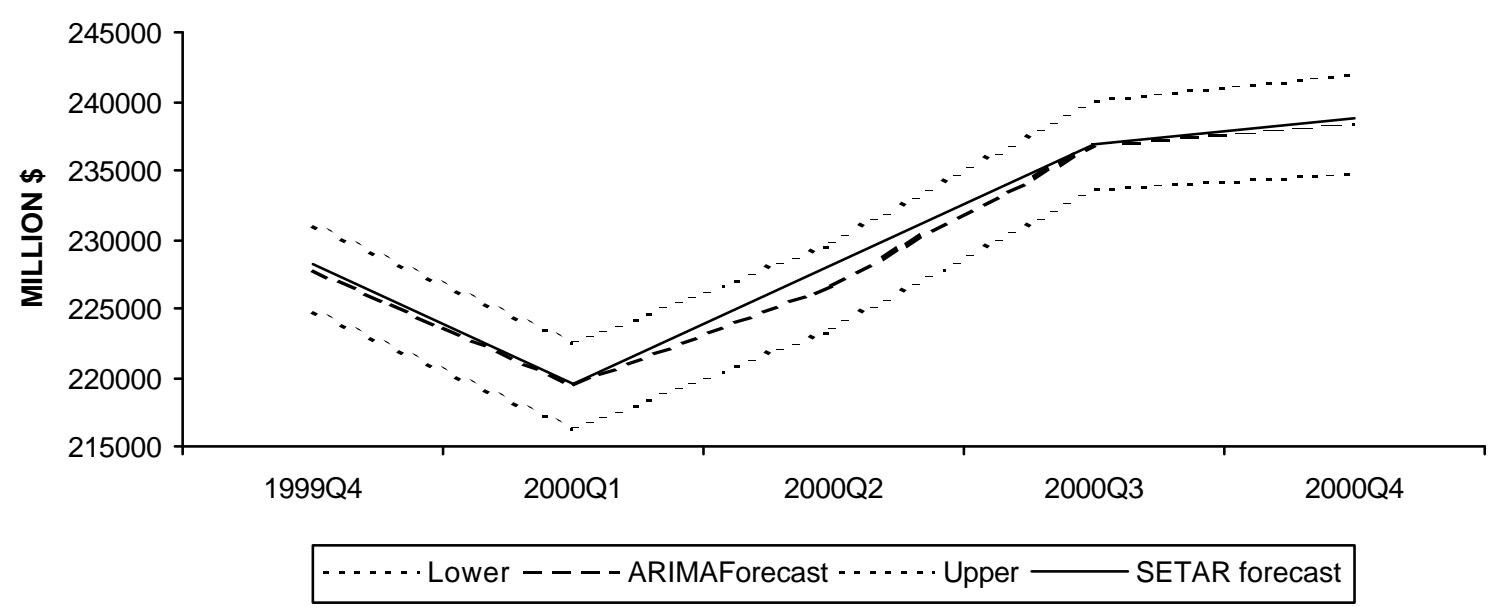

Although it is quite complicated to get the forecasting interval for the SETAR model using both forecasting methods, we can pursue some of the information about the SETAR forecasting interval indirectly. Figure 5 describes the $95 \%$ forecasting interval of the ARMA model by using the 1-step-ahead forecasting method and also the locations of the forecasted values for both models using the same forecasting method. It is easy to see that the out-of-sample 1-step-ahead forecasting values of the SETAR model all locate within the one-step-ahead 95\% confidence forecasting intervals of ARIMA model respectively. This indicates that the $95 \%$ confidence forecasting intervals of both models by using the 1-step-ahead forecasting method overlap each other to some degree. Taking their standard deviation into the consideration, we could conclude that the forecasting intervals of the two models are not differently from each other. As a result, the 1-step-ahead forecasting performances of both models are not significantly different from each other.

\section{Conclusions}

In this paper we consider the nonlinear features of Canadian real GDP data by using a SETAR model and we study the out-of-sample forecasting performance of this model by comparing it with a standard linear ARIMA model. Two forecast evaluation techniques are employed in this paper to compare the relative forecast performances of the two 
models. These two forecasting methods are: the multi-step-ahead forecast and the 1-stepahead forecast. In the terms of the forecasting results under the two forecasting methods, both models appear to be satisfactory because the forecasting MAPEs and RSMPEs for both models are all under $2 \%$. However, the SETAR model performs better than the ARIMA model not only with respect to within-sample fit, but also with respect to the outof-sample forecasting performance. As for the two forecasting methods we have applied in this paper, they both could give us good forecasting results, but in real life, the multistep-ahead forecasting tends to be more practical. 


\section{References:}

Bacon, D. W., and Watts, D. G. (1971). Estimating the transition between two intersecting lines, Biometrika, 58, 525-534.

Clements, M. P., and Smith, J. (1997). The performance of alternative forecasting methods for SETAR models, International Journal of Forecasting, 13, 463-475.

Clements, M. P., and Krolzig, H. M. (1998). A comparison of the forecast performance of Markov-switching and threshold autoregressive models of U.S. GNP, Econometrics Journal, 1, C47-75.

Clements, M. P., and Smith, J. (1999). A Monte Carlo study of the forecasting performance of empirical SETAR models, Journal of Applied Econometrics, 14, 123-141.

Clements, M. P., Franses, P. H. and Smith, J. (1999). On SETAR non-linearity and forecasting, Econometric Institute Report 141, Erasmus University Rotterdam, Econometric Institute.

Christiano, L. J., and Eichenbaum, M. (1992). Current real business cycle theories and aggregate labor market fluctuations, American Economic Review, 86, 430-450.

Chappell, D., Padmore, J., Mistry, P., and Ellis, C. (1996). A threshold model for the French franc/deutschmark exchange rate, Journal of Forecasting, 15, 155-164.

De Gooijer J.G., and Kumar, K. (1992). Some recent development in non-linear time series modeling, testing and forecasting, International Journal of Forecasting, 8, $135-156$.

Dacco, R., and Satchell, S. (1999). Why do regime-switching models forecast so badly? Journal of Forecasting, 18, 1-16.

Diebold, F.X., and Mariano, R.S. (1995). Comparing predictive accuracy, Journal of Business and Economic Statistics, 13, 253-263.

Franses, P.H., and van Dijk, D. (2000). Non-Linear Time Series Models in Empirical Finance, Cambridge University Press, Cambridge.

Gallegati, M., and Mignacca, D. (1995). Nonlinearities in business cycle: SETAR model and G7 industrial production data, Applied Economics Letters, 2, 422-427. 
Lin, J.L., and Granger, C.W.J (1994). Forecasting from non-linear models in practice, Journal of Forecasting, 13, 1-9.

Kräger, H., and Kugler, P. (1993). Non-linearities in foreign exchange markets: A different perspective, Journal of International Money and Finance, 12, 195-208.

Montgomery A.L., Zarnowitz V., Tsay R., Tiao C (1998). Modeling non-linearity in the U.S. unemployment rate with unobserved component models, Technical Report, University of Chicago, Graduate School of Business.

Peel, D. A., and Byers, J. D. (1994). Cross country evidence on nonlinearity in industrial production between the wars, Applied Economics Letters, 1, 77-80.

Peel, D. A., and Speight A.E.H. (1994). Testing for non-linear dependence in inter-war exchange rates, Weltwirtschaftliches Archive, 2, 391-417

Peel, D. A., and Speight, A. E. H. (1998). Threshold nonlinearities in output: Some international evidence, Applied Economics, 30, 323-333.

Potter, S. M. (1995). A nonlinear approach to U.S. GNP, Journal of Applied Econometriecs, 10, 109-125.

Pindyck, R. S., and Rubinfeld, D. L. (1991). Econometric Models and Economic Forecasts, McGraw-Hill, New York.

Rothman P. (1998). Forecasting asymmetric unemployment rates, Working Paper, East Carolina University, Dept. of Economics.

SHAZAM (1997). SHAZAM Econometrics Computer Program, Version 8.0: User's Reference Manual, McGraw-Hill, New York.

Sowell, F. (1992). Modeling long-run behaviour with the fractional ARIMA model, Journal of Monetary Economics, 29, 277-302.

Teräsvirta, T. (1998). Modeling economic relationships with smooth transition regressions. In A. Ullah and D. E. A. Giles (eds.), Handbook of Applied Economic Statistics, Marcel Dekker, New York, 507-552.

Tiao, G. C., and Tsay, R.S. (1994). Some advances in non-linear and adaptive modeling in time series, Journal of Forecasting, 13,109-131.

Tong, H. (1978). On a threshold model, in Chen, C.H.(ed.), Pattern Recognition and Signal Processing, Sijhoff and Noordoff, Amsterdam.

Tong, H. (1983). Threshold Models in Non-Linear Time Series Analysis, SpringerVerlag, New York. 
Tong, H. (1995). Non-linear Time Series. A Dynamical System Approach, Clarendon Press, Oxford.

Tong, H., and Lim, K. S. (1980). Threshold autoregression, limit cycles and cyclical data, Journal of The Royal Statistical Society, B 42, 245-292. 


\section{Footnotes:}

1 The usefulness of linear models is usually judged by their predictive ability, and such models are usually used as a benchmark for econometric models in forecast comparisons. However the choice of the ARIMA model here is somewhat arbitrary.

2 For details, see Franses and van Dijk (2000).

3 We considered both first differenced data and the original level data. We found that the SETAR model doesn't fit the first differenced data very well and has very bad forecasting performance. Accordingly, the level of real GDP is used in our comparative analysis.

4 The ARIMA model is estimated using the SHAZAM (1997) econometrics package. We also wrote a program in SHAZAM code to estimate our SETAR model. 